\title{
Die Aktivität der Lipoproteinlipase und der Postheparinesterase bei Störungen des Fettstoffwechsels
}

\author{
Von A. Štork, L. KučERová und E. FABIAN \\ Aus der I. Medizinischen Klinik der Karls-Universität, Prag, Tschechoslowakei (Direktor: Prof. Dr. V. Hoenig, Dr.Sc.)
}

(Der Schriftleitung zugegangen am 6. Dezember 1963)

\begin{abstract}
Die Lipoproteinlipase- und Postheparinesterase-Aktivität ist bei einer Gruppe von Hepatikern, Nephritikern, Kranken mit idiopathischer Hyperlipämie und Diabetikern untersucht und mit der Aktivität dieser Enzyme bei einer Kontrollgruppe gesunder Personen verglichen worden.

Die LPL-Aktivität war bei Nephritikern und Patienten mit idiopathischer Hyperlipämie bedeutend vermindert, während bei Diabetikern die Aktivität dieses Enzyms signifikant erhöht war. Bei Hepatikern war die Aktivität dieses Enzyms normal. Die PHE-Aktivität war bei Hepatikern bedeutend vermindert, dagegen bei Nephritikern signifikant erhöht. Bei Hyperlipämikern und Diabetikern war die PHE-Aktivität zwar erhöht, jedoch ștatistisch nicht signifikant. Die möglichen Ursachen der Aktivitätsänderung dieser Enzyme wurden bei allen untersuchten Gruppen besprochen.

The activity of lipoprotein lipase and postheparin esterase in a group of patients with hepatitis, nephritis, idiopathic hyperlipaemia and diabetes was studied and compared with the activity of these enzymes in a control group of healthy persons:

LPL-activity was significantly lower in patients with nephritis and patients with idiopathic hyperlipaemia, but significantly higher in diabetics. It was normal in patients with hepatitis. PHE-activity was significantly lower in patients with hepatitis, but significantly higher in patients with nephritis. In hyperlipaemia and diabetes, there was an increased PHE-activity, but it was not statistically significant. Possible causes of these changes in enzyme activity in each group are discussed.
\end{abstract}

Der gestörte Fettstoffwechsel kann die Begleiterscheinung einer Reihe von Krankheiten sein. In unserer Arbeit bemühen wir uns um eine Klärung der Rolle, die die veränderte Aktivität der Lipoproteinlipase („LPL") und der Postheparinesterase („PHE“) bei einer Störung des Fettstoffwechsels bei einer chronischen Leberkrankheit, bei der Glomerulonephirits, der idiopatischen Hyperlipämie und bei Diabetes mellitus spielen könnte. Diese beiden Fermente sind ein Bestandteil einer durch Heparin aktivierten Klärungsreaktion und können ihrem Ursprung nach $(1,2)$, der Spezifität des Substrats $(3,4)$ und den unterschiedlichen Inhibitoren $(5,6)$, unterschieden werden.

Die LPL wird durch Heparin aus verschiedenen Geweben zumeist aus dem Fettgewebe (7-10) freigesetzt. Ihr Substrat sind plasmatische Triglyceride (11-15), die einen Bestandteil der Lipoproteine im Blutplasma bilden. Es wird mit Natriumchlorid, Protamin und Heparin in Überschuß inhibiert $(2,6,16)$. Dagegen entsteht die PHE in der Leber (1). Ihr spezifisches Substrat in vivo ist bisher unbekannt, in vitro führt sie zur Spaltung verschiedener Ester (17-20). Die angeführten Inhibitoren der LPL wirken auf sie nicht ein. - Beide Enzyme können gut unterschieden werden, bei der Bestimmung ihrer Aktivität in vitro, bei der die LPL das lipämische Plasma, oder die künstliche Fettemulsion, die vorher mit Plasmaproteinen inkubiert wurde, spaltet, wogegen die PHE die Esterbindung der Tweene $(17,18)$, des Äthylbutyrats $(19,20)$ und anderer Ester spaltet.

Wir untersuchten 7 Patienten mit chronischem Leberleiden, 7 Kranke mit Glomerulonephritis, 10 Patienten mit idiopatischer Hyperlipämie und 10 Diabetes-Kranke. Als Kontrollgruppe wurden 10 gesunde Personen untersucht.

\section{Methodik}

Bei allen Untersuchten wurde Blut nach zwölfstündigem Fasten abgenommen, mit derselben Nadel $50 \mathrm{mg}$ Heparin verabreicht und nach 10 Minuten von dem anderen Arm eine weitere Blutabnahme durchgeführt. Als Antigerinnungsmittel wurden 2 Tropfen Heparin verwendet. Die Blutproben wurden sofort verarbeitet. Beim Postheparinplasma wurde die Aktivität der PHE nach KATZ (17) unter Verwendung von Tween 80 als Substrat und die Aktivität der LPL auf die Weise bestimmt, daß $2 \mathrm{~m} l$ Plasma verdünnt 1:5 mit physiol. Kochsalzlösung innerhalb von 2 Stunden bei $37^{\circ}$ mit $2 \mathrm{ml}$ gleich verdünnten lipämischen Plasma einer gesunden Person inkubiert wurde. Der Unterschied zwischen der Extinktion vor und nach der Inkubation in Prozenten ausgedrückt war dann das Maß der Aktivität der LPL. In gleicher Weise ist beim Vorheparinplasma vorgegangen worden, das keine Klärungsaktivität aùfwies. Das Ablesen wurde mit Hilfe eines Klett-Summerson Photokolorimeters mit einem Filter von $540 \mathrm{~m} \mu$ durchgeführt.

\section{Ergebnisse}

Von den 7 untersuchten Hepatikern (Tab. 1), im Durchschnittsalter von 49 Jahren, waren 3 Männer und 4 Frauen. Der Durchschnittswert der PHE-Aktivität war 1,43 Mikromol (0-4,55 $\mu \mathrm{Mol})$, der Durchschnittswert der LPL-Aktivität 29,34\% (20,50-36,90\%).

Tab. 1

Aktivität der Postheparinesterase und der Lipoproteinlipase bei Leberkranken

\begin{tabular}{cccccc}
\hline $\begin{array}{c}\text { Patient } \\
\text { Nr. }\end{array}$ & Geschlecht & $\begin{array}{c}\text { Alter } \\
\text { (Jahre) }\end{array}$ & Diagnose & $\begin{array}{c}\text { Aktivität } \\
\text { der PHE } \\
\text { in } \\
\mu \mathrm{Mol} \text { der }\end{array}$ & $\begin{array}{c}\text { der LPL } \\
\text { in vitro } \\
\text { in \% }\end{array}$ \\
\hline 1 & männl. & 56 & Cirrhosis hepatis & 0 & 36,90 \\
2 & weibl. & 72 & Cirrhosis hepatis & 0,20 & 23,40 \\
3 & männl. & 46 & Cirrhosis hepatis & 0 & 20,50 \\
4 & männl. & 31 & Hepatitis chron. & 0 & 37,40 \\
5 & weibl. & 49 & Hepatitis chron. & 2,00 & 30,10 \\
6 & weibl. & 54 & Cirrhosis biliaris & 3,30 & 27,40 \\
7 & weibl. & 41 & Hepatitis chron. & 4,55 & 29,90 \\
\hline
\end{tabular}


Von 7 Kranken mit Nierenleiden (Tab. 2) waren 5 Männer und 2 Frauen im Durchschnittsalter von 30 Jahren. Der Durchschnittswert der PHE-Aktivität war 5,93 $\mu \mathrm{Mol}$ $(4,55-8,30 \mu \mathrm{Mol})$ und der Durchschnittswert der LPLAktivität $12,2 \%(2,38-29,6 \%)$.

Tab. 2

Aktivität der Postheparinesterase und der Lipoproteinlipase bei Nierenkranken

\begin{tabular}{|c|c|c|c|c|c|}
\hline $\begin{array}{l}\text { Pat. } \\
\text { Nr. }\end{array}$ & $\begin{array}{c}\text { Ge- } \\
\text { schlecht }\end{array}$ & $\begin{array}{c}\text { Alter } \\
\text { (Jahre) }\end{array}$ & Diagnose & $\begin{array}{c}\text { Aktivität } \\
\text { der PHE } \\
\text { in } \\
\mu \mathrm{Mol}\end{array}$ & $\begin{array}{c}\text { Aktivität } \\
\text { der LPL } \\
\text { in vitro } \\
\text { in } \%\end{array}$ \\
\hline
\end{tabular}

\begin{tabular}{llrlrr}
\hline 1 & männl. & 42 & $\begin{array}{c}\text { Glomerulonephritis } \\
\text { chron. }\end{array}$ & 6,70 & 25,40 \\
2 & männl. & 35 & $\begin{array}{c}\text { Glomerulonephritis } \\
\text { chron. }\end{array}$ & 8,30 & 2,38 \\
4 & männl. & 27 & $\begin{array}{c}\text { Glomerulonephritis } \\
\text { chron. }\end{array}$ & 6,55 & 27,60 \\
5 & männl. & 22 & $\begin{array}{c}\text { Glomerulonephritis } \\
\text { subacuta }\end{array}$ & 5,20 & 9,30 \\
6 & weibl. & 14 & $\begin{array}{c}\text { Nephrotisches } \\
\text { Syndrom }\end{array}$ & 5,20 & 9,30 \\
7 & männl. & 24 & $\begin{array}{c}\text { Glomerulonephritis } \\
\text { chron. }\end{array}$ & 4,55 & 25,10 \\
\hline & $\quad \begin{array}{c}\text { Nephrotisches } \\
\text { Syndrom }\end{array}$ & 5,55 & 29,60 \\
\hline
\end{tabular}

Bei 10 an idiopatischer Hyperlipämie kranken Männern im Durchschnittsalter von 45 Jahren (Tab. 3 ) war der Durchschnittswert der PHE-Aktivität 4,57 $\mu \mathrm{Mol}(2,45$ bis $7,05 \mu \mathrm{Mol}$ ), der Wert der LPL-Aktivität $10,93 \%$ (0-23,20\%).

Tab. 3

Aktivität der Postheparinesterase und der Lipoproteinlipase bei IHL-Kranken

\begin{tabular}{ccccc}
\hline $\begin{array}{c}\text { Patient } \\
\text { Nr. }\end{array}$ & Geschlecht & $\begin{array}{c}\text { Alter } \\
\text { (Jahre) }\end{array}$ & $\begin{array}{c}\text { Aktivität } \\
\text { der PHE } \\
\text { in } \\
\mu \mathrm{Mol}\end{array}$ & $\begin{array}{c}\text { Aktivität } \\
\text { der LPL } \\
\text { in vitro } \\
\text { in \% }\end{array}$ \\
\hline 1 & männl. & 50 & 3,05 & 7,15 \\
2 & männl. & 36 & 5,55 & 11,75 \\
3 & männl. & 43 & 2,45 & 10,50 \\
4 & männl. & 48 & 3,25 & 23,20 \\
5 & männl. & 30 & 3,65 & 21,90 \\
6 & männl. & 45 & 5,00 & 9,00 \\
7 & männl. & 51 & 4,80 & 5,26 \\
8 & männl. & 56 & 7,05 & 0 \\
9 & männl. & 58 & 5,95 & 12,20 \\
10 & männl. & 41 & 4,90 & 8,30 \\
\hline
\end{tabular}

Bei Diabetikern (Tab. 4), 6 Männern und Frauen - im Durchschnittsalter von 50 Jahren - mit Krankheitsdauer des Diabetes durchschnittlich 14 Jahre, war die durchschnittliche PHE-Aktivität 4,14 $\mu \mathrm{Mol}(0,15-7,40$ $\mu \mathrm{Mol}$ ), die der Lipoproteinlipase $48,73 \%$ (30,76 bis 69,67\%).

Die Kontrollgruppe bildeten 10 gesunde Personen (Tab. 5), 5 Männer und 5 Frauen mit einem Durchschnittsalter von $36 \mathrm{Jahren}$, bei denen der durchschnittliche PHE-
Wert $3,69 \mu \mathrm{Mol}$ betrug $(1,4-5,2 \mu \mathrm{Mol})$, der LPLAktivität 33,86\% (20,30-58,68\%).

Die Durchschnittswerte der einzelnen Gruppen sind, um einen leichteren Úberblick $z u$ ermöglichen, in der Tabelle 6 zusammengefaßt.

Bei der statistischen Wertung mit H-Test zwischen den einzelnen Gruppen der Patienten und der Kontrollgruppen wurde festgestellt, daß die PHE-Aktivität bei den Hepatikern auf der $5 \%$ Bedeutungsebene $(\mathrm{H}=$ $5,48>3,84$ ), signifikant niedriger ist. Bei den Nephri-

Tab. 4

Aktivität der Postheparinesterase und der Lipoproteinlipase bei Diabetikern

\section{Dauer der Täglicher}

Pat. Ge- Alter Diabetes- Insulin- Aktivität Aktivität

Nr. schlecht (Jahre) erkrankung bedarf der PHE der LPL

\begin{tabular}{rlrrrrr} 
& & & $\begin{array}{c}\text { (in } \\
\text { Jahren) }\end{array}$ & $\begin{array}{c}\text { (Ein- } \\
\text { heiten) }\end{array}$ & $\begin{array}{c}\text { in } \\
\mu \text { Mol }\end{array}$ & $\begin{array}{c}\text { in vitro } \\
\text { in \% }\end{array}$ \\
\hline 1 & männl. & 58 & 31 & 80 & 3,05 & 32,80 \\
2 & männl. & 65 & 21 & 90 & 1,18 & 69,67 \\
3 & männl. & 29 & 10 & 80 & 7,13 & 65,41 \\
4 & männl. & 53 & 10 & 60 & 2,10 & 32,80 \\
5 & männl. & 52 & 8 & 106 & 5,65 & 42,22 \\
6 & männl. & 68 & 7 & 40 & 5,80 & 54,54 \\
7 & weibl. & 30 & 17 & 44 & 7,40 & 46,80 \\
8 & weibl. & 55 & 3 & 60 & 0,15 & 60,00 \\
9 & weibl. & 70 & 27 & 60 & 5,80 & 30,76 \\
10 & weibl. & 22 & 15 & 90 & 3,15 & 52,30 \\
\hline
\end{tabular}

Tab. 5

Aktivität der Postheparinesterase und der Lipoproteinlipase bei der Kontrollgruppe

\begin{tabular}{rlccc}
\hline $\begin{array}{c}\text { Patient } \\
\text { Nr. }\end{array}$ & Geschlecht & $\begin{array}{c}\text { Alter } \\
\text { (Jahre) }\end{array}$ & $\begin{array}{c}\text { Aktivität } \\
\text { der PHE } \\
\text { in } \\
\mu \mathrm{Mol}\end{array}$ & $\begin{array}{c}\text { Aktivität } \\
\text { der LPL } \\
\text { in vitro } \\
\text { in \% }\end{array}$ \\
\hline 1 & weibl. & 16 & 5,20 & 34,40 \\
2 & männl. & 27 & 5,20 & 20,30 \\
3 & weibl. & 34 & 3,55 & 25,30 \\
4 & weibl. & 30 & 3,60 & 30,70 \\
5 & männl. & 27 & 1,40 & 34,90 \\
6 & männl. & 25 & 3,95 & 34,40 \\
7 & männl. & 32 & 2,05 & 32,70 \\
8 & männl. & 76 & 4,60 & 33,00 \\
9 & weibl. & 26 & 4,20 & 34,30 \\
10 & weibl. & 25 & 3,15 & 58,60 \\
\hline
\end{tabular}

Tab. 6

Durchschnittswerte der Aktivität der Postheparinesterase (PHE) und der Lipoproteinlipase (LPL) bei den untersuchten Gruppen

\begin{tabular}{lcccc}
\hline Gruppe & $\begin{array}{c}\text { Zahl } \\
\text { der } \\
\text { Unter- } \\
\text { suchten }\end{array}$ & $\begin{array}{c}\text { Durch- } \\
\text { schnitts- } \\
\text { alter } \\
\text { (Jahre) }\end{array}$ & $\begin{array}{c}\text { Aktivität } \\
\text { der PHE } \\
\text { in } \\
\mu \text { Mol }\end{array}$ & $\begin{array}{c}\text { Aktivität } \\
\text { der LPL } \\
\text { in vitro } \\
\text { in \% }\end{array}$ \\
\hline Leberkranke & 7 & 49.85 & 1,43 & 29,34 \\
Nierenkranke & 7 & 30,80 & 5,93 & 12,20 \\
Hyperlipämiekranke & 10 & 45,80 & 4,57 & 10,93 \\
Diabetiker & 10 & 50,20 & 4,14 & 48,73 \\
Kontrollgruppe & 10 & 36,80 & 3,69 & 33,86 \\
\hline
\end{tabular}


tikern war sie wesentlich erhöht und dies sogar auf der $1 \%$ Ebene $(\mathrm{H}=8,00>6,63)$. Bei den übrigen Gruppen unterscheidet sich die Aktivität dieses Enzyms statistisch nicht von der Kontrollgruppe.

Was die LPL-Aktivität anbelangt, ist sie auf der $1 \%$ Ebene bei Nephritikern $(\mathrm{H}=7,46>6,63)$ und bei Patienten mit idiopatischer Hyperlipämie $(\mathrm{H}=13,1>$ 6,63 ) herabgesetzt. Bei den Diabetikern ist die Aktivität dieses Enzyms auf der 5\% Bedeutungsebene signifikant erhöht $(H=4,10>3,84)$. Bei den Hepatikern unterscheidet sich die LPL-Aktivität nur unwesentlich von der dieses Enzyms bei der Kontrollgruppe.

\section{Diskussion}

\section{Lipoproteinlipase}

Die LPL ist bei Hepatikern nach den angeführten Ergebnissen genau so aktiv wie bei der Kontrollgruppe, obwohl laut Literaturangaben $(21-25)$ bewiesen ist, daß dieses Ferment in der Leber abgebaut wird. Diese Desintegrationstätigkeit der Leber sinkt parallel mit der Schädigung des Leberparenchyms (23), so daß der Unterschied in der Aktivität dieses Ferments im Arterienblut und im Blut der Lebervenen indirekt proportional zu der Funktionsfähigkeit der Leber steht, woraus sich ergibt, daß bei schwerer Leberschädigung seine Aktivität erhöht ist $(21,22,24)$. Im Hinblick darauf, daß sämtliche Patienten bei denen wir die Aktivität dieses Ferments bestimmen, entweder gut kompensierte Cirrhotiker oder Kranke mit chronischer Hepatitis waren, wogegen es sich in den oben erwähnten Arbeiten um Fälle schwerer Leberschädigung handelte, könnte angenommen werden, daß bei unseren Kranken die Funktionsfähigkeit des Leberparenchyms noch hinreichend im Verhältnis zur Desintegration der LPL stand.

Die wesentliche Verminderung der LPL-Aktivität bei Nephritikern können wir einerseits durch die Hypalbuminämie bei diesen Patienten erklären, die bekanntlich (26-32) zu einer Erhöhung der Lipämie führt und dies sowohl beim Tierexperiment als auch beim Menschen. Es hat den Anschein, daß hier überdies der Verlust irgendeines der Kofaktoren durch die Nieren
(31) oder die Änderung der Zusammensetzung der Plasmaproteine $(26,33)$ mitwirken. Es hat sich gezeigt, daß bei einer Nephritis die Fähigkeit der Albumine, durch Klärungsreaktion freigewordene Fettsäuren (34) zu binden, herabgesetzt ist.

Die Verminderung der LPL-Aktivität bei der idiopatischen Hyperlipämie ist komplexer Natur. Wie wir bereits in einer unserer früheren Publikationen bewiesen haben (35), beteiligt sich an der Störung der ganzen Klärungsreaktion bei dieser Erkrankung sowohl die verminderte Spaltbarkeit der Chylomikronen und der Mangel an Eiweißakzeptoren der freigewordenen Fettsäuren einerseits als auch die Aktivitätssenkung der LPL oder Inhibitorenanwesenheit andererseits.

Bemerkenswert ist der Befund der erhöhten Aktivität dieses Ferments bei Diabetikern. Diese Aktivitätserhöhung im Plasma der Kranken mit kompensiertem Diabetes steht im Widersprụch zu dem bekannten Befund der Fettmobilisierung (36-39) und Erniedrigung der LPL-Aktivität im Fettgew̌ebe $(2,8)$ bei dem dekompensierten Diabetes.

\section{Postheparinesterase}

Die Senkung der PHE-Aktivität bei Hepatikern wurde auch von anderen Autoren gefunden (40-43) und ist ein Beweis, daß sie von der Leber herrührt (1). Aus den erzielten Ergebnissen, obwohl die Gruppe der Kranken klein ist, geht hervor, daß die Aktivitätssenkung dieses Enzyms im ganzen parallel mit der sinkenden Leberfunktionsfähigkeit verläuft.

Die überraschende Erhöhung der PHE-Aktivität bei Nephritikern kann auf zweierlei Art erklärt werden. Entweder durch die Mitbeteiligung der Nieren - sei es in der Inaktivierung, oder in der Aussonderung dieses Enzyms - oder dadurch, daß das Heparin wegen der verminderten LPL-Aktivität nicht ausgenützt werden konnte und in größerer Menge für die Aktivierung der PHE zur Verfügung stand. Als Beweis für diese Schlußfolgerung könnte die niedrige LPL-Aktivität nicht nur bei einer Nierenerkrankung, sondern auch bei der idiopathischen Hyperlipämie dienen. Bei dieser Krankheit ist nämlich die PHE-Aktivität ebenfalls erhöht, wenn auch statistisch nicht signifikant.

\section{Literatur}

1. Páv, J., J. Wenkeová und E. Kunn, Cas. fysiol. 9, 41 (1960). 2. Páv, J. und J. Wenkeoví, Tukový metabolismus a lipoproteinavá lipáza. Thomayerova sbirka 415, SZdN, Praha (1962). 3. Páv, J., J. Wenkeová und E. Kunn, Cas. lék. česk. 100, 273 (1961). - 4. Skořepa, J., B. Procházka und H. Todorovi Cová, Sborn. lék. 62, 33 (1960). - 5. SkoŘEpA, J. und S. NovÁk, Nature (London) 182, 117 (1958). - 6. SxoŘkpa, J., Enzymologie tukové přeměny. Thomayerova sbirka 397, SZdN, Praha (1960). - 7. GrafNetTer, D. und T. ZeMPLÉNYI, Hoppe-Seyler's Z. physiol. Chem. 316, 218 (1959). - 8. Wenkeová, J., B. Mosinger und J. PÁv, Cas. fysiol. 9, 482 (1960). - 9. ZEMPLÉNYI, T. und D. GrafNETter, Čas. lék. česk. 97, 638 (1958). - 10. ZEMPLÉNYr, T. und D. Grafnetter, Cas. lék. česk. 98, 97 (1959). - 11. KorN, E. D., Circulation 10, 591 (1954). - 12. KoRN, E. D., Science 120, 399 (1954). - 13. Sprtzer, J. J., Heparin and Lipid Trans-

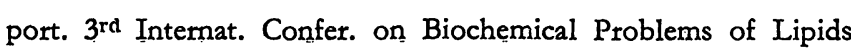
$26^{\text {th }}$ to $28^{\text {th }}$ July, 1956. - 14. Swank, R. L., Amer. J. Pathol. 164, 798 (1951). - 15. Swank, R. L. und S. W. Lewr, Amer. J. Physiol. 171, 208 (1952). - 16. Brown, R. K., E. Boyle, jr. und Ch. B. ANfinsen jr., Federat. Proc. 11, 18 (1952). - 17. Katz, S., J. appl. Physiol. 10, 519 (1957). - 18. PÁv, J., Z. Placer und Z. Roubal, Cas. lék. česk. 97, 620 (1958). - 19. SkořepA, J., Vnitř. lék. 2, 394 (1956). - 20. Skorepa, J. und H. Todorovi ČovÁ, Cas. lék. česk. 95, 289 (1956). - 21. BAKER, S. P., H. LeVINE, L. Turner und A. Dubin, Proc. Soc. exp. Biol. 99, 670 (1958). 22. Baker, S. P., P. P. Foá, L. Turner und A. Dubin, Proc. Soc. exp. Biol. Med. 101, 464 (1959). - 23. ConNor, W. E. und J. W. Eckstern, J. Clin. Invest. 38,1746 (1959). - 24. Constantinides, P., Y. So. und F. R. C. Johnstone, Proc. Soc. exp. Biol. Med. 100, 262 (1959). - 25. Spitzer, J. A. und J. J. Spitzer, 
Amer. J. Physiol. 185, 18 (1956). - 26. KuČerová, L. und A. ŚTORK, Cas. lék. česk. 101, 618 (1962). - 27. KuČEROVÁ, L. und A. Štork, Cas. lék. česk. 101, 1137 (1962). - 28. Pezold, F. A., Klin. Wschr. 37, 132 (1959). - 29. Pezord, F. A., Dtsch. Arch. klin. Med. 205, 640 (1959). - 30. Rosenman, R. H., M. FriedMAN und S. O. Byers, J. Clin. Invest. 35, 522 (1956). - 31. Rosenman, R. H. und M. Friedman, J. Clin. Invest. 36, 700 (1957). - 32. Rosenman, R. H., S. O. Byers und M. Friedman, J. Clin. Invest. 36, 1558 (1957). - 33. Monkhouse, F. C. und R. G. MacKneson, Canad. J. Biochem. Physiol. 36, 1065 (1958). - 34. Axenfeld, H., Wien. klin. Wschr. 68, 296 (1956). - 35. Kučeroví, L., A. Śtork und E. Fabian, Cas. lék. česk. - im Druck. - 36. Hallgren, B., S. Stenhagen, A. Svanborg und
L. Svennerholm, J. Clin. Invest. 39, 1424 (1960). - 37. Hrsich, P. F., Ann. int. Med. 41, 546 (1956). - 38. Thannhauser, S. J., Lipidoses. Diseases of the intracellular lipid metabolism. Grune and Stratton, New York and London (1958). - 39. ZöLLNER, N. und S. J. Thannhauser, Thannhausers Lehrbuch des Stoffwechsels und der Stoffwechselkrankheiten. Georg Thieme Verlag, Stuttgart (1957). - 40. Krondr, A., H. Vavrínková, C. MrchaLEC und andere, Cas. gastroenterol. 14, 129 (1960). - 41. PLACER, Z., A. KRONDL und Z. Slabochová, Cas. gastroenterol. 13, 145 (1959). - 42. Skorera, J., Acta Univ. Carol. Med. 4, 181 (1959). - 43. STork, A. und L. Kučerová, Čas. lék. česk. 101, 833 (1962).
Doz. Dr. A. Stork

I. Med. Klinik der Karls-Universität

Prag 2, Ǔ nemocnice 2

\title{
Zur Kenntnis der Lipase-Bestimmung nach Willstätter
}

\author{
Von C. G. vom Bruck \\ Aus den Forschungslaboratorien der Nordmark-Werke Hamburg, Werk Uetersen.
}

(Der Schriftleitung zugegangen am 16. Mai 1964)

\begin{abstract}
Bei der Bestimmung der Pankreaslipase nach der Methode von WILLSTÄTTER in der Modifikation von VoGEL und LAEVERENZ wurde die Fettsäurebestimmungsmethode verbessert. Die Fettsäuren werden aus dem angesäuerten Spaltansatz durch Benzol extrahiert und photometrisch als Kupfersalz bestimmt. Mit dieser Methode wurde die Abhängigkeit der Olivenölspaltung von Lipasemenge erneut untersucht und eine von der von WrLLSTÄTrER angegebenen abweichende Dosiswirkungskurve angegeben.
\end{abstract}

In the estimation of pancreatic lipase, according to WILLSTÄTTER, modified by Vogel and LAEVERENZ, the fatty acid determination was improved. The fatty acids are extracted from the acidified hydrolysis mixture with benzene and determined photometrically as the copper salt. With this method, the dependence of the hydrolysis of olive oil on the amount of lipase was reinvestigated; a dose-activity curve, different from that of WILLSTÄTTER, is given.

Die von WILLSTÄTter, WALDSCHMIDT-LEITZ und MEMMEN (1) 1923 veröffentlichte Lipase-Bestimmungsmethode wurde 1935 von Vogel und Laeverenz (2) durch Erhöhung der Albumin- und CalciumchloridKonzentration und Zufügen von Natriumoleat zur Sicherung einer gleichmäßigen Emulsionsbildung verbessert. Diese modifizierte WILLSTÄTtER-Methode ist seitdem vielfach zur Ermittlung der Lipasegehalte von Fermentpräparaten verwandt worden, besonders zur Beurteilung technisch hergestellter Pankreatine.

Neben der WiLLSTärTER-Methode ist eine große Zahl anderer Lipase-Bestimmungsmethoden angegeben worden (vgl. 3), die sich nach Verwendung von wasserlöslichen, bzw. praktisch wasserunlöslichen Estern als Substrat, in zwei Gruppen einteilen lassen. Nach Untersuchungen von DesNuELLE und seiner Schule (4) ist die Pankreas-Lipase eine Spezial-Esterase, die bevorzugt an der Grenzfläche Ölphase/Wassexphase wirkt, $d . h_{\text {., da die }}$ wasserunlöslichen Fette, wie z. B. Olivenöl, ideale Substrate für die Pankreas-Lipase sind. Die Ester niedriger Fettsäuren werden zwar durch Lipasen, aber auch durch einfache Esterasen gespalten, was sich nachteilig auf die Spezifität der betreffenden Methoden auswirkt. Die wasserlöslichen Ester der höheren Fettsäuren, wie z. B. Produkte der Tween-Gruppe, werden nach Dessuelre (4) durch Pankreaslipase nicht gespalten, sondern durch ein diese begleitendes Ferment "Tweenase", deren Abtrennung von der Pankreaslipase möglich war.

Im folgenden sind zwei Methoden beschrieben, mit denen die aus Olivenöl im Vogel-Laeverenz-Spalt- ansatz freigesetzten Fettsäuren genauer als nach der von WILLSTÄTTER angegebenen Titrationsmethode bestimmt werden können; außerdem wird eine neue Lipase-Spaltungskurve bei Verwendung von Pankreatin angegeben.

Bei Extraktion mit Benzol und 5 Tropfen konz. Salzsäure nach den Angaben von LASO-WASEM (5) werden nur etwa 50\% der Fettsäuren erfaßt, obwohl der pH-Wert der Mischung 0,8-0,9 beträgt. Durch Zusatz von $1 \mathrm{ml}$ konz. Salzsäure ( $\mathrm{pH}$ etwa 0,1 ) werden bei einmaliger Extraktion mit Benzol $85 \pm 2 \%$ (Standardabweichung; $n=10$ ) der Fettsäuren erfaßt, wie durch Zugabe von 600-2400 $\mu \mathrm{M}$ Ölsäure ermittelt wurde. - Die Extraktion mittels Isopropylalkohol/n-Heptan/Wasser nach DoLe (6), Chloroform/Methanol/Wasser nach FolCH (7) und BLIGH und Dyer (8) bzw. Isopropylalkohol/Benzol/Wasser ergaben keine besseren Ausbeuten. Der Fettsäuregehalt wurde durch Titration mit methanolischer Natronlauge und dem Mischindikator nach TrwarI (9) bestimmt.

Noch einfacher können jedoch die Fettsäuren photometrisch nach einem Verfahren, das von BAKER ${ }^{1}$ ) (10) angegeben wurde, bestimmt werden. Die benzolische Lösung der Fettsäuren wird mit einer Kupferacetatlösung geschüttelt, wodurch sich benzollösliche, grüngefärbte Kupfersalze der Fettsäuren bilden. Deren Ex-

1) Herrn Prof. Wolf und Herrn Dr. Sarau möchte ich auch an dieser Stelle für den Literaturhinweis und methodische Angaben herzlich danken. 